Bioética

\section{UTILIZANDO BEM OS ÍNDICES PROGNÓSTICOS}

Os índices prognósticos têm sido cada vez mais utilizados em pacientes em unidades de terapia intensiva (UTI) para avaliação da qualidade de uma determinada UTI, para comparação entre UTls, para a randomização de pacientes em protocolos de estudo, etc. A prática de se usar índices para prever o prognóstico de um paciente individualmente deve ser vista com extrema cautela. Ridley AS, em artigo recente, revisa de uma maneira muito simples as bases matemáticas em que os índices se apoiam e quais seus possíveis pontos de erro e suas limitações. Inicialmente, devese ter em mente que os índices fornecem uma probabilidade e não uma predição absoluta de que um determinado evento ocorra (no caso óbito ou sobrevida). No desenvolvimento dos índices, o grupo inicial de pacientes é formado segundo critérios bem definidos de inclusão e de exclusão, portanto a aplicação de um determinado índice para um grupo de pacientes, ou para um paciente individualmente, com características diferentes do grupo inicial não é recomendável. Outros eventos igualmente importantes, como é a qualidade de vida, não são preditos. Os parâmetros que entraram no desenvolvimento do índice e a maneira como foram coletados, manual ou automaticamente, devem ser os mesmos a serem utilizados para um paciente individual. Por exemplo: a presença de sedação pode impedir uma avaliação adequada do estado de consciência de um paciente e conseqüentemente diminuir a acurácia do cálculo do seu risco de morte. Ridley também analisa os problemas que podem surgir dos métodos utilizados na validação dos índices e o seu poder de discriminação. Um bom índice deve apresentar uma boa discriminação e uma boa calibração, duas qualidades que em geral não são concomitantes nos índices de caráter genérico. A utilização de índices para se determinar a futilidade de um tratamento para um paciente individual deve ser feita com cautela e não deve, de maneira nenhuma, substituir o julgamento clínico.

\section{Comentário}

Deve-se sempre ter em mente que os índices prognósticos são somente mais uma ferramenta a ser utilizada pelo médico quando se deparar com uma decisão difícil de limitação de tratamento ou na alocação de recursos escassos como são as vagas em UTIs. A melhor maneira de bem utilizar os índices prognósticos é conhecer as suas limitações.

Regina C. R. M. Abdulkader Referência

Ridley AS. Uncertainty and scoring systems, Anaesthesia 2002; 57: 76I-7.

\section{Clinica Crringiea}

\section{DOPPLER ENDOSCÓPICO E} VARIZES GASTRO-ESOFAGIANAS

É consenso que para avaliar risco de sangramento por varizes gastro-esofagianas é essencial o conhecimento de sua fisiopatologia, e para tanto Hino e colaboradores realizaram este estudo da morfologia e da hemodinâmica da veia gástrica esquerda (VGE) através da Doppler endoscópico colorido.

$\mathrm{Na}$ população estudada havia doentes com varizes de fino, médio e grosso calibres. Sendo identificado que o fluxo sangüíneo hepatofugal no tronco da VGE aumentou com o progressivo desenvolvimento do calibre das varizes, mas não correspondeu ao aumento do diâmetro da VGE.

Assim como comprovaram que o aumento progressivo do calibre das varizes correspondeu a um predomínio do fluxo para o ramo anterior da VGE em detrimento ao ramo posterior. Não se identificou, apesar do aumento das varizes, relação com o desenvolvimento do calibre das veias colaterais paraesofagianas, mas correspondeu ao aumento do calibre das veias perfurantes e de sua detecção ao exame.

Os autores concluíram que a velocidade do fluxo sangüíneonotroncoda VGE, emseus ramos enasveias perfurantes, poderegular osuprimento do fluxo sangüíneo para as varizes esofagianas e contribuir para o seu desenvolvimento.

\section{Comentário}

Aspecto de suma importância no acompanhamento do paciente hepatopata é a perfeita noção do estado da hemodinâmica portal. É importante 0 estudo sistemático para esclarecimento da instalação do regime de hipertensão portal, da fisiopatologia dos sangramentos e de evidências que demonstrem risco eminente de hemorragia.

Talvez, mais importante que a identificação de valores isolados, seriam as mensurações repetidas, ou seja, controles seqüêciais para traçar o perfil evolutivo da doença.

Darcy lisbão Moreira de Carvalho

Pedro luiz Squilacci Leme

Referência

Hino S, Kahutani K, Kuramochi A, Ikeda K, Uchiyama $Y$, Sumiyama K, et al. Hemodynamic assessment of the left gastric vein in patients with esophageal varices with color Doppler EUS: Factors affecting development of esophageal varices gastroeintist Endosc 2002; 55(4): 512-7.

\section{Clínica Médica \\ A OBESIDADE E A INSUFICIÊNCIA CARDÍACA}

A insuficiência cardíaca (IC) tornou-se um dos grandes problemas de Saúde Pública do mundo moderno. Apesar dos avanços terapêuticos, a morbidade e a mortalidade por insuficiência cardíaca permanece muito alta, sendo necessário desta forma a sua prevenção, que deve ter um enfoque prioritário.

A obesidade extrema é reconhecida, há anos, como um fator de risco para insuficiência cardíaca. Dados do estudo de Framingham mostraram, com base na análise de 588 I pessoas, com idade média de 55 anos, sendo $54 \%$ mulheres, que o aumento do índice de massa corpórea (IMC) em uma unidade aumentaria o risco de apresentar IC em $5 \%$ para os homens e em $7 \%$ para as mulheres. Quando se compara a evolução dos obesos (IMC de 30 ou mais) com os normais (IMC entre 18,5 e 24,9) os obesos apresentaram o dobro de chance de apresentar descompensação cardíaca, sendo este risco 2, 12 para as mulheres e I,90 para os homens. 
Este estudo populacional, de análise de uma comunidade, documentou que pacientes com aumento do IMC apresentam uma maior chance de desenvolver IC. Dado o aumento da prevalência da obesidade, especialmente nos Estados Unidos, mas também em certas regiões do Brasil, estratégias promovendo a busca do peso ótimo podem reduzir a epidemia de IC dos nossos dias.

Se a obesidade é um fator de risco para o desenvolvimento da IC,éinteressanteentretanto notar que ela confere uma possível proteção quanto à mortalidade aos pacientes portadores de IC. Numa coorte de 1203 pacientes com disfunção ventricular (FE média de 0,22) e IC pode-se observar que aqueles com maior IMC (IMC > 3I) apresentaram uma mortalidade menor do que os três grupos com IMC mais baixo (<20,7; entre 20,7 e 27,7 e entre 27,3 e 3I).

Os dados deste estudo mostraram que nos pacientes com IC avançada a obesidade não estaria associada a um aumento de mortalidade e que ela parece oferecer um prognóstico mais favorável.

\section{Comentário}

A obesidade é um fator de risco para a populaçãa sadia, com os obesos apresentando pelo menos duas vezes mais quadro clínico de IC do que os sem aumento de peso, mas nos portadores de $I C$ o fato de o paciente não estar emagrecido está associado a um melhor prognóstico. Este resultado decorreria de uma menor estimulação neurohormonal, incluindo uma menor elevação do fator de necrose tumoral-alfa, fato que confere aos pacientes uma melhor evolução, uma vez que a estimulação neurohormonal aumentada é um importante determinante de progressão da doença e do prognóstico.

Observamos nestes estudos os dois aspectos extremos de uma doença, pois para prevenir a IC, controlar o excesso de peso é benéfico e com a IC instalada, evitar o emagrecimento reduz mortalidade. É importante, no entanto, ressaltar que os dados destes dois estudos são fundamentados em análise de população e não foram desenhados prospectivamente para verificar se a perda do peso realmente reduziria o risco de $I C$ e se a prevenção da perda de peso nos portadores de IC reduziria a sua mortalidade.

Antonio Carlos Pereira Barretto
Referências

I. Kenchaiah S, Evans JC, Levy D, Wilson PW, Benjamin EJ, Larson MG, etal. Obesity and the risk of heart failure. N Engl J Med 2002; 347: 305-13.

2. Horwich TB, Fonarow GC, Hamilton MA, Mac Lellan WR, Woo MA, Tellisch JH. The relationship between obesity and mortality in patients with heart failure. J Am Coll Cardiol 200I; 38: 789-95.

\section{Economia da Sacide \\ CUSTO-EFICÁCIA DOS AGENTES DE SAÚDE COMUNITÁRIOS CONTRA \\ A TUBERCULOSE EM BANGLADESH}

O objetivo deste trabalho foi o de comparar o custo-eficácia do programa contra a tuberculose, dirigido pelo Comitê para o Progresso Rural em Bangladesh (Brac), com a utilização de agentes de saúde comunitário, com o programa governamental contra a tuberculose que não utiliza os referidos agentes.

O período compreendido foi entre juIho de 1996 a junho de 1997. Os dados de TB e custos foram coletados de um subdistrito do BRAC, e um subdistrito do governo a $100 \mathrm{~km}$ de Dakar, a capital, com semelhança sócio-demográfica, ambas similiares de grande maioria de outros subdistritos.

O custo-eficácia foi calculado pela divisão entre 0 custo por atividade e as diferentes medidas de eficácia no período de um ano.

O custo por paciente curado foi calculado dividindo o número de pacientes curados no total do custo do sistema de saúde. O custo global por paciente curado foi calculado pela soma de custo do sistema de saúde e custo do paciente.

A análise de custo anual foi de US\$ 7,35I para BRAC e US\$10,697 para 0 governamental. Nas zonas controladas pelo BRAC e pelo Governo foi identificado um total de 186 e 185 doentes de tuberculose, respectivamente; ao longo do ano as taxas de cura foram de $84 \%$ e de $82 \%$ entre os pacientes considerados positivos. No entanto, o custo por pacientes curado foi de US\$ 64 nas zonas do BRAC e de US\$ 96 na zona da administração.

Os autores concluíram que o programa governamental foi $50 \%$ mais caro em igualdade de resultados nos dois programas.

A participação dos agentes comunitários resultam em uma opção, mas adequada da análise do custo-eficácia nas zonas rurais de Bangladesh. Concluíram também que, com o mesmo orçamento do programa BRAC, é possível curar três doentes de TB para cada dois curados no programa governamental.

\section{Comentário}

A tuberculose tem sido um grande problema nos países subdesenvolvidos. Como pudemos observar no trabalho apresentado, os custos do programa governamental para pacientes com TB foram $50 \%$ mais caros do que os da BRAC, o que representa que para três doentes tratados pela BRAC e curados, 0 programa governamental consegue tratar e curar somente dois pacientes.

A inferência é que, em geral, os programas governamentais tendem a ser mais custosos pelos procedimentos legais exigidos para contratação de pessoas, compra de medicamentos, etc. Por outro lado, os programas com agentes comunitários têm a facilidade de conhecer melhor a comunidade onde atuam, bem como suas necessidades. Por essa razão, suas chances de sucesso aumentam e com custo menor.

Vitória Kedy Cornetta Referência

Islam A, WakaiS, Ishikawa N, Cowdhury AMR, Vaughan P. Cost- effectiveness of community health workers in tuberculoses control in Bangladesh. Bull World Health Organ 2002; 80 (6): 445-50.

\section{Emergêneia e Medicina Toutensiva \\ A VIA DE SINALIZAÇÃo CD40/ CD40L NA SEPSE GRAVE}

A sepse e sua expressão mais grave, o choque séptico, são as principais causas de mortalidade nas unidades de terapia intensiva 